\title{
Comparative Analysis of Image Enhancement Technique for Hyperspectral Palmprint Images
}

\author{
Anita G. Khandizod \\ Department of Computer Science and Information \\ Technology, Dr. Babasaheb Ambedkar \\ Marathwada University, Aurangabad
}

\author{
R.R. Deshmukh \\ Department of Computer Science and Information \\ Technology, Dr. Babasaheb Ambedkar \\ Marathwada University, Aurangabad
}

\begin{abstract}
Image Enhancement is one of the most important and difficult techniques, also the first stage in the pre-processing of images which have to be subjected to image recognition algorithms. The goal of image enhancement is to improve the quality of images. Palmprint image quality is an important factor in the performance of hyperspectral palmprint recognition system. To the best of knowledge there is no evidence of work specifically directed towards image enhancement techniques on hyperspectral palm print images. In this paper different thirteen types of image enhancement techniques are compared based on image quality measure (subjective and objective), subjective image quality measure based on histogram and objective quality measure is based on mean square error (MSE), Peak signal to noise ratio (PSNR), Normalized cross correlation (NK), Average difference (AD), Structural content (SC), Maximum difference (MD), Normalized absolute error (NAE). Such a comparison would be useful in determining the best suited image enhancement method for hyperspectral palmprint. Median filter gives good performance as compare to other image enhancement techniques. The performance of different thirteen image enhancement techniques are tested on PolyU hyperspectral palmprint database. The comparative results are tabled.
\end{abstract}

\section{General Terms}

Image processing

\section{Keywords}

Image Enhancement, image quality measure, Spatial and frequency Domain, Image restoration,

\section{INTRODUCTION}

Image enhancement plays an important role in digital image processing [1]. When any image is captured through device, the clarity of image can be affected by optic, weather, sensor limitations, and different illumination conditions. These may lead to image information lose or poor quality. The main aim of image enhancement is to get more details of an image that is hidden in an image and highlight the useful information. In this process input low quality images are passes and the output high quality images are used for specific application.

In day to day life there is frequent need in identifying and verifying person correctly, obviously high accuracy is required during the identification [2].

The work on hyperspectral palmprint recognition is quite limited in literature. Hyperspectral imaging offers new opportunities for palmprint recognition, hyperspectral images are rich in information, processing the hyperspectral data poses several challenging task. Hyperspectral imaging may be useful for increasing the accuracy because of its high dimensionality.

In this paper total different thirteen image enhancement techniques are used which is categorized in three types first spatial domain enhancement including negative Image, logarithmic transformation, power law transformation, second frequency domain enhancement like Butterworth low pass filter, Butterworth high pass filter, Gaussian Low pass filter, Gaussian High Pass filter, and third image restoration such as contrast limited adaptive histogram equalization (CLAHE), Adjusted Image, Median filter, 2D order statics, Adaptive filter, Decorrelation Stretch.

It is often necessary to measure the quality of the different thirteen image enhancement techniques this can be done by using, "Image quality measure", which categorized into two types subjective and objective image quality measure [3]. In subjective quality measure the quality of image is evaluated by using histogram based on human observance while objective quality measure based on statistical parameter. However, Subjective quality measure is time consuming process, inconvenient and expensive, too slow for practical usage, and varies from person to person; therefore it is important to use objective image quality measure. Objective image quality is measured using seven different types of quality measure like Mean square error (MSE), peak signal to noise ratio (PSNR), maximum difference (MD), Normalized cross correlation (NK), Normalized absolute error (NAE), absolute difference (AD) [4]

The organization of this paper is given as follow Section 2 discusses the description of PolyU Hyperspectral palmprint database; Section 3 discusses the development of various image enhancement techniques. Section 4 reviews the image quality measure Section 5 result analysis and comparison; section 6 discussed the conclusion of the paper.

\section{HYPHERSPECTRAL PALMPRINT DATABASE}

A large hyperspectral palmprint database is developed by the Biometric Research Center at Department of Computing, the Hong Kong Polytechnic University. Images were collected from 190 individuals. The age distribution was from 20 to 60 years old. The samples have been collected in two separate sessions. The average time interval between the two sessions was around 1 month. In each session, the subject was asked to provide around seven cubes of each of his/her left and right palms, so the database contains 5240 images for each band from 380 different palms and the size of each palmprint is $128 * 128$ pixels [5]. 


\section{IMAGE ENHANCEMENT}

The reason for doing image enhancement are higlighting interesting detail in images, removing noise and making images more visually appealing, and find out the best image enhancement technique based on image quality measure for hyperspectral palmprint images. The Different image enhancement technique as fallows

\subsection{Spatial Domain Enhancement}

Spatial domain enhancement method is based on direct manipulation of pixels in an image.

$$
\mathrm{g}(\mathrm{x}, \mathrm{y})=\mathrm{T}[\mathrm{f}(\mathrm{x}, \mathrm{y})]
$$

Spatial domain image enhancement techniques are categorized into three types as fallows.

\subsubsection{Negative Image}

Negative images are useful for enhancing white or grey detail embedded in dark regions of an image; each value of the input image is subtracted from the L-1 and mapped onto the output image.

\subsubsection{Logarithmic Transformation}

The log transformation maps a narrow range of low input grey level values into a wider range of output values [6]. The inverse $\log$ transformation performs the opposite transformation; they are especially useful for bringing out detail in an image.

\subsubsection{Power law Transformation}

Map a narrow range of dark input values into a wider range of input values or vice versa, where $\mathrm{c}$ and $\gamma$ are positive constants.

$$
\mathrm{s}=\mathrm{c} * \mathrm{r} ?
$$

\subsection{Frequency Domain Enhancement}

Frequency domain techniques are based on the manipulation of the orthogonal transform of the image rather than the image itself. The basic model for filtering is:

$$
\mathrm{G}(\mathrm{u}, \mathrm{v})=\mathrm{H}(\mathrm{u}, \mathrm{v}) \mathrm{F}(\mathrm{u}, \mathrm{v})
$$

Frequency domain image enhancement techniques are categorized into four types as fallows.

\subsubsection{Butterworth low pass filter}

The transfer function of a Butterworth low pass filter of order $\mathrm{n}$ with cut off frequency at distance D0 from the origin is defined as

$$
H(u, v)=\frac{1}{1+\left[D(u, v) / D_{0}\right]^{2 n}}
$$

\subsubsection{Gaussian Low pass filter}

The transfer function of a Gaussian low pass filter is defined as:

$$
H(u, v)=e^{-D^{2}(u, v) / 2 D_{0}^{2}}
$$

\subsubsection{Butterworth Highpass filter}

The Butterworth high pass filter is given as:

$$
H(u, v)=\frac{1}{1+\left[D_{0} / D(u, v)\right]^{2 n}}
$$

\subsubsection{Gaussian High pass filter}

The Gaussian high pass filter is given as:

$$
H(u, v)=1-e^{-D^{2}(u, v) / 2 D_{0}^{2}}
$$

\subsection{Image Restoration}

It deals with improving the appearance of an image. The Image is corrected using different image restoration technique with improving the appearance of an image.

\subsubsection{Contrast limited Adaptive Histogram Equalization}

Contrast Limited Adaptive Histogram Equalization (CLAHE) is a generalization of Adaptive Histogram Equalization and used to prevent the problem of noise. By using this method the hidden features of the image more visible. The method has three parameters.

\subsubsection{Median filter}

As the name clear the median filter is statistics method. In median filter the median of the pixel the replace the pixel by median of the gray levels in their neighborhood of that pixel [7]. Median filter is best because of its excellent noise reduction ability from the images. By the filtering it keeps the edges while removing the noise.

\subsubsection{Adjusted Image}

Adjust image maps the intensity values in image to new values in such that $1 \%$ of data is saturated at low and high intensities of input image. This increases the contrast of the output image.

\subsubsection{D-Order-statistic Filter}

2D order statistic filter working with large domain matrices that do not contain any zero-valued elements, ordfilt 2 can achieve higher performance if input image is in an integer data format (uint8, int8, uint16, int16). The gain in speed is larger for uint 8 and int 8 than for the 16-bit data types.

\subsubsection{D-Adaptive Filter}

Adaptive Wiener method based on statistics estimated from a local neighborhood of each pixel, and the objective is to find an estimate $\mathrm{f}^{\wedge}$ of the uncorrupted image $f$ such that the mean square error between them is minimized. This error measure is given by

$$
\mathrm{e} 2=\mathrm{E}\left\{\left(\mathrm{f}-\mathrm{f}^{\wedge}\right) 2\right\}
$$

\subsubsection{Decorrelation Stretch}

A decorrelation stretch is a linear, pixel-wise operation in which the specific parameters depend on the values of actual and desired (target) image statistics. The vector a containing the value of a given pixel in each band of the input image $A$ is transformed into the corresponding pixel $\mathrm{b}$ in output image $\mathrm{B}$ as follows:

$$
\mathrm{b}=\mathrm{T} *(\mathrm{a}-\mathrm{m})+\mathrm{m} \_ \text {target } .
$$

\section{IMAGE QUALITY MEASURE}

Image Quality measure are essential for most image processing application, is a characteristic of an image that measures the perceived image degradation compared to an ideal or perfect image [8]. There are two types of image quality measure.

\subsection{Subjective Image Quality}

Subjective measurements are the result of human experts providing their opinion of the image quality but the result vary person to person. In subjective measurements the quality of image is decided by using histogram. Histogram manipulation can be used effectively for image enhancement technique. 
Histograms are simple to calculate in software and also lend themselves to economic hardware implementations, thus making them a popular tool for real-time image processing. Histogram is the graphical representation of gray level values $(0-255)$ of image against frequency. Histogram is categorized into four basic gray-level characteristics: dark, light, low contrast, and high contrast [9].

In Dark image the components of the histogram are concentrated on the low (nearer 0) side of the gray scale. Similarly, the components of the histogram of the bright image are biased toward the high side of the gray scale. In low contrast the component of the histogram will be centered toward the middle of the gray scale. Histogram in the highcontrast image cover a broad range of the gray scale and, further, that the distribution of pixels is not too far from uniform, with very few vertical lines being much higher than the others. High contrast image gives the good quality.

\subsection{Objective Image Quality}

Objective measurements are performed with mathematical algorithms. The objective Image Quality measure (IQM) can be classified into full-reference, reduced-reference and noreference. FR criteria are also a function of the original image which is assumed to be free from distortions (called the "reference image"). RR criteria require a partial knowledge of the reference image (this knowledge is called the "reduced reference"). At last, NR criteria don't have any information about the reference image [10].

The simplest and most widely used full-reference quality metric is the mean squared error (MSE), computed by averaging the squared intensity differences of distorted and reference image pixels, along with the related quantity of Peak Signal-to-Noise Ratio (PSNR). These are appealing because they are simple to calculate, have clear physical meanings, and are mathematically convenient in the context of optimization.

\section{RESULTS AND ANALYSIS}

The image of hyperspectral palmprint is of high resolution and useful data are presents in a spectral band. To enhance the quality of palmprint image, different thirteen types of image enhancement technique are used, and the quality of the image is decided by using image quality measure.

As show in fig 1 the histogram component of negative and logarithmic transformation is low contrast because the histogram component will be centered toward the middle of the gray scale, so the quality of the image is poor, while power law transformation having dark image, In case of adjust image and Decorrelation stretch the component of the histogram some people think like the image low contrast or may be light image so the decision varies from person to person, because result by subjective image quality measure are human observer and time consuming, objective quality measure are used to find out the best image enhancement technique for hyperspectral palmprint recognition. Hyperspectral PolyU database contain 69 spectral bands (4201100 ) but the first ten and the last five spectra are removed due to the low quality of images [8], therefore 54 spectral bands (520-1050), are used for experiment.

\begin{tabular}{|c|c|c|}
\hline $\begin{array}{c}\text { Spatial Domain } \\
\text { Enhancement }\end{array}$ & Enhanced Image & $\begin{array}{c}\text { Histogram of } \\
\text { Enhanced Image }\end{array}$ \\
\hline $\begin{array}{l}\text { Negative } \\
\text { Image }\end{array}$ & & \\
\hline $\begin{array}{c}\text { Logarithmic } \\
\text { Transformation }\end{array}$ & & \\
\hline $\begin{array}{c}\text { Power Law } \\
\text { Transformation }\end{array}$ & & \\
\hline & & Dark mage \\
\hline
\end{tabular}

Fig 1: Spatial Domain Enhanced Image and its histogram

\begin{tabular}{|c|c|c|}
\hline $\begin{array}{l}\text { Frequency } \\
\text { Domain } \\
\text { Enhancement }\end{array}$ & $\begin{array}{l}\text { Enhanced } \\
\text { Image }\end{array}$ & $\begin{array}{l}\text { Histogram of } \\
\text { Enhanced Image }\end{array}$ \\
\hline $\begin{array}{l}\text { Gaussian } \\
\text { Lowpass Filter }\end{array}$ & & \\
\hline $\begin{array}{l}\text { Butterworth } \\
\text { Highpass filter }\end{array}$ & & \\
\hline $\begin{array}{l}\text { Gaussian } \\
\text { Highpass Filter }\end{array}$ & $\begin{array}{l}x \\
4 \\
4\end{array}$ & sine \\
\hline
\end{tabular}

Fig 2: Frequency Domain Enhanced Image and its histogram

Table 1 shows the seven objective image quality measure and there parameter based on that, the quality of image depends on the lower and higher score value.

Table 1. Image Quality Parameter

\begin{tabular}{|c|c|c|}
\hline $\begin{array}{l}\text { Sr. } \\
\text { No }\end{array}$ & $\begin{array}{l}\text { IMAGE QUALITY } \\
\text { MEASURE }\end{array}$ & QUALITY OF IMAGE \\
\hline 1 & Mean Square Error & $\begin{array}{c}\text { Lower MSE Value provide } \\
\text { Lower Quality }\end{array}$ \\
\hline 2 & $\begin{array}{l}\text { Peak Signal to noise } \\
\text { Ratio }\end{array}$ & $\begin{array}{l}\text { Higher PSNR value } \\
\text { provide higher Quality }\end{array}$ \\
\hline 3 & $\begin{array}{l}\text { Normalized Cross } \\
\text { correlation }\end{array}$ & $\begin{array}{l}\text { Score Value range of one } \\
\text { higher quality }\end{array}$ \\
\hline 4 & Average Difference & $\begin{array}{l}\text { Higher MD value provide } \\
\text { lower Quality }\end{array}$ \\
\hline 5 & Structural Content & $\begin{array}{c}\text { lower SC Value provide } \\
\text { higher quality }\end{array}$ \\
\hline 6 & Maximum difference & $\begin{array}{l}\text { Higher MD value provide } \\
\text { higher Quality }\end{array}$ \\
\hline 7 & $\begin{array}{c}\text { Normalized Absolute } \\
\text { Error }\end{array}$ & $\begin{array}{l}\text { large value of NAE means } \\
\text { that image is poor quality }\end{array}$ \\
\hline
\end{tabular}


As shown in table 2 the image quality assessment of 54 spectral bands and their objective quality measure, for median filter image enhancement technique. Similarly for remaining image enhancement technique objective quality measure are calculated. As show in table 3 the median filter image enhancement technique gives good result as compare to other image enhancement technique, the MSE value for median filter is 14.6, $\mathrm{PSNR}=36.6, \mathrm{NK}=0.99$ and so on, therefore according to the table 1 median filter gives good quality of image which is used for the feature extraction and further process of hyperspectral palmprint images.

Table 2. Comparison of Objective Image Quality Metrics for Median filter

\begin{tabular}{|c|c|c|c|c|c|c|c|}
\hline \multicolumn{8}{|c|}{ Original Image+ Median filter } \\
\hline Spectral Band & MSE & PSNR & NK & AD & SC & MD & NAE \\
\hline 520 & 31.96 & 33.08 & 0.997 & 0.15 & 1.003 & 151 & 0.024 \\
\hline 530 & 15.78 & 36.15 & 0.999 & 0.07 & 1.001 & 157 & 0.016 \\
\hline 540 & 27.64 & 33.72 & 0.998 & 0.02 & 1.001 & 166 & 0.025 \\
\hline 550 & 13.22 & 36.92 & 0.999 & 0.08 & 1.002 & 155 & 0.015 \\
\hline 560 & 18.03 & 35.57 & 0.998 & 0.07 & 1.002 & 159 & 0.02 \\
\hline $\mathbf{5 7 0}$ & 13.7 & 36.76 & 0.999 & 0.05 & 1.001 & 153 & 0.016 \\
\hline 580 & 50.75 & 31.08 & 0.996 & 0.18 & 1.006 & 138 & 0.036 \\
\hline 590 & 18.15 & 35.54 & 0.998 & 0.12 & 1.003 & 142 & 0.021 \\
\hline 600 & 16.5 & 35.96 & 0.998 & 0.12 & 1.003 & 140 & 0.02 \\
\hline 610 & 12.79 & 37.06 & 0.999 & 0.11 & 1.002 & 142 & 0.017 \\
\hline 620 & 14.43 & 36.54 & 0.998 & 0.1 & 1.002 & 126 & 0.019 \\
\hline 630 & 15.65 & 36.19 & 0.998 & 0.12 & 1.003 & 114 & 0.02 \\
\hline 640 & 9.455 & 38.37 & 0.999 & 0.09 & 1.002 & 137 & 0.014 \\
\hline 650 & 10.63 & 37.87 & 0.999 & 0.11 & 1.002 & 141 & 0.015 \\
\hline 660 & 13.17 & 36.94 & 0.998 & 0.15 & 1.003 & 140 & 0.018 \\
\hline 670 & 9.397 & 38.4 & 0.999 & 0.13 & 1.002 & 132 & 0.014 \\
\hline 680 & 10.45 & 37.94 & 0.999 & 1 & 1.002 & 134 & 0.015 \\
\hline 690 & 11.74 & 37.43 & 0.999 & 0.15 & 1.003 & 130 & 0.017 \\
\hline 700 & 11.53 & 37.51 & 0.998 & 0.17 & 1.003 & 132 & 0.016 \\
\hline 710 & 9.417 & 38.39 & 0.998 & 0.15 & 1.003 & 136 & 0.014 \\
\hline 720 & 10.43 & 37.95 & 0.998 & 0.16 & 1.003 & 126 & 0.016 \\
\hline 730 & 9.441 & 38.38 & 0.999 & 0.14 & 1.002 & 135 & 0.014 \\
\hline 740 & 13.11 & 36.95 & 0.998 & 0.18 & 1.003 & 114 & 0.019 \\
\hline 750 & 10.83 & 37.78 & 0.998 & 0.16 & 1.003 & 126 & 0.016 \\
\hline 760 & 17.96 & 35.59 & 0.998 & 0.18 & 1.004 & 85 & 0.022 \\
\hline 770 & 20.61 & 34.99 & 0.997 & 0.2 & 1.004 & 93 & 0.024 \\
\hline 780 & 13.88 & 36.71 & 0.998 & 0.14 & 1.003 & 105 & 0.019 \\
\hline 790 & 16.78 & 35.88 & 0.997 & 0.24 & 1.005 & 105 & 0.022 \\
\hline 800 & 15.25 & 36.3 & 0.998 & 0.17 & 1.003 & 130 & 0.02 \\
\hline 810 & 11.75 & 37.43 & 0.998 & 0.17 & 1.003 & 125 & 0.017 \\
\hline 820 & 17.63 & 35.67 & 0.997 & 0.21 & 1.004 & 99 & 0.022 \\
\hline 830 & 19.72 & 35.18 & 0.997 & 0.23 & 1.005 & 97 & 0.024 \\
\hline 840 & 21.59 & 34.79 & 0.997 & 0.23 & 1.005 & 89 & 0.025 \\
\hline 850 & 24.34 & 34.27 & 0.997 & 0.26 & 1.005 & 82 & 0.027 \\
\hline 860 & 7.469 & 39.4 & 0.999 & 0.1 & 1.002 & 173 & 0.011 \\
\hline 870 & 10.98 & 37.73 & 0.998 & 0.18 & 1.003 & 128 & 0.016 \\
\hline 880 & 12.19 & 37.27 & 0.998 & 0.17 & 1.003 & 126 & 0.018 \\
\hline 890 & 13.25 & 36.91 & 0.998 & 0.19 & 1.004 & 112 & 0.019 \\
\hline 900 & 15.03 & 36.36 & 0.998 & 0.2 & 1.004 & 103 & 0.02 \\
\hline 910 & 14.62 & 36.48 & 0.998 & 0.18 & 1.003 & 96 & 0.02 \\
\hline 920 & 13.59 & 36.8 & 0.998 & 0.17 & 1.003 & 95 & 0.019 \\
\hline 930 & 7.264 & 39.52 & 0.999 & 0.09 & 1.001 & 167 & 0.011 \\
\hline 940 & 8.902 & 38.64 & 0.999 & 0.13 & 1.002 & 117 & 0.014 \\
\hline 950 & 8.299 & 38.94 & 0.999 & 0.13 & 1.002 & 120 & 0.014 \\
\hline 960 & 8.06 & 39.07 & 0.999 & 0.11 & 1.002 & 122 & 0.013 \\
\hline 970 & 8.259 & 38.96 & 0.999 & 0.12 & 1.002 & 123 & 0.014 \\
\hline 980 & 11.92 & 37.37 & 0.998 & 0.15 & 1.003 & 123 & 0.018 \\
\hline 990 & 12.03 & 37.33 & 0.998 & 0.14 & 1.003 & 125 & 0.018 \\
\hline 1000 & 12.1 & 37.3 & 0.998 & 0.13 & 1.003 & 127 & 0.018 \\
\hline 1010 & 14.04 & 36.66 & 0.998 & 0.15 & 1.003 & 128 & 0.019 \\
\hline 1020 & 10.53 & 37.91 & 0.999 & 0.09 & 1.002 & 146 & 0.016 \\
\hline 1030 & 13.49 & 36.83 & 0.998 & 0.14 & 1.003 & 126 & 0.018 \\
\hline 1040 & 14.95 & 36.38 & 0.999 & 0.1 & 1.002 & 131 & 0.019 \\
\hline 1050 & 17.91 & 35.6 & 0.998 & 0.18 & 1.004 & 129 & 0.021 \\
\hline MEAN & 14.68 & 36.79 & 0.998 & 0.14 & 0.258 & 173.9 & 0.018 \\
\hline
\end{tabular}


Table 3.Tabular comparison of image enhancement technique

\begin{tabular}{|c|l|c|c|c|c|c|c|c|}
\hline & \multicolumn{7}{|c|}{ Objective Image Quality Measure } \\
\hline $\begin{array}{c}\text { Sr. } \\
\text { No }\end{array}$ & $\begin{array}{l}\text { Image enhancement } \\
\text { Technique }\end{array}$ & MSE & PSNR & NK & AD & SC & MD & NAE \\
\hline 1 & Median Filter & 14.67723 & 36.79143 & 0.998148 & 0.144341 & 0.258116 & 173.9074 & 0.018386 \\
\hline 2 & CLAHE & 427.1094 & 21.88987 & 1.086433 & -11.0797 & 0.83706 & 48.40741 & 0.131588 \\
\hline 3 & Adjust Image & 3292.278 & 12.97137 & 1.353912 & -40.2397 & 0.525462 & 74.7963 & 0.375438 \\
\hline 4 & Order-statistic & 36.02105 & 33.04606 & 1.027262 & -3.76181 & 0.946508 & 14.59259 & 0.034942 \\
\hline 5 & Adaptive filter & 17.42081 & 36.1917 & 0.997404 & 0.193351 & 1.004173 & 19.96296 & 0.024507 \\
\hline 6 & Decorrelation stretch & 1747.315 & 15.83629 & 1.015908 & 3.938905 & 0.917725 & 86 & 0.269973 \\
\hline 7 & Butterworth Lowpass & 3878.773 & 12.24386 & 0.525608 & 62.24485 & 3.5422 & 64 & 0.486288 \\
\hline 8 & Butterworth Highpass & 6813.176 & 9.810128 & 0.557271 & 55.20869 & 2.219829 & 126.9074 & 0.568169 \\
\hline 9 & Gaussian Lowpass & 16528.97 & 5.948344 & 1.94471 & -127 & 1.002839 & 68.92593 & 0.992187 \\
\hline 10 & Gaussian Highpass & 11539.28 & 7.521595 & 1.48391 & -66.4453 & 0.379608 & 166.4074 & 0.767809 \\
\hline 11 & Negative Image & 1600.96 & 16.087 & 0.94471 & 1.00012 & 1.015429 & 117.1481 & 0.254807 \\
\hline 12 & Logarithmic Transformation & 651.5633 & 19.99125 & 0.804218 & 24.62499 & 1.544981 & 46.44444 & 0.192383 \\
\hline 13 & Power low Transformation & 16239.4 & 6.025113 & 0.01651 & 126.16 & 1883.501 & 164.7778 & 0.985625 \\
\hline & Min Value & 14.67723 & 5.948344 & 0.01651 & -127 & 0.258116 & 14.59259 & 0.018386 \\
\hline & Max Value & 16528.97 & 36.79143 & 1.94471 & 126.16 & 1883.501 & 173.9074 & 0.992187 \\
\hline
\end{tabular}

High Oualitv Image

\section{CONCLUSION}

In this paper, 13 image enhancement techniques have been applied to improve the quality of hyperspectral palmprint image, to decide the quality image quality measure like subjective and objective image quality measure are used subjective analysis can't be relied on because it varies from person to person on the basis of their image perception and visual assessments and also time consuming, therefore subjected the enhanced image to objective quality measure like MSE, PSNR, NK, AD, SC, MD, NAE, on the basis of them different thirteen image enhancement technique are compared. As per finding 2D median filter is the best image enhancement technique for hyperspectral palmprint image as compare to the other technique. In future, In future feature extraction technique like 2D PCA and 2D LDAs are applied on best image enhanced technique 2D median filter.

\section{ACKNOWLEDGMENTS}

1. Thanks to the Hong Kong Polytechnic University, for sharing their database, (PolyU Hyperspectral palmprint Database)

2. This project is under UGC Rajiv Gandhi National Fellowship F1-17.1/2011-12/RGNF-SC-MAH-9445, sanction at department of CS \& IT, Dr. Babasaheb Ambedkar Marathwada University, Aurangabad.

\section{REFERENCES}

[1] Youhei Terai, Tomio Goto, "Color Image Enhancement," Proceedings of IEEE, pp-392-393, Iligan City, 2009.

[2] Anita G. Khandizod, R. R. Deshmukh, "Analysis and Feature Extraction using Wavelet based Image Fusions for Multispectral Palmprint Recognition", International Journal of Enhanced Research in Management \& Computer Applications, ISSN: 2319-7471 Vol. 3 Issue 3, March-2014, pp: (57-64), Impact Factor: 1.147, Available online at: www.erpublications.com.

[3] Anita G. Khandizod, R.R.Deshnukh, Ramesh.R.Manza, "Wavelet-based image fusion and quality assessment of
Poor Oualitv Image

Multispectral Palmprint Recognition", 2nd National Conference on Computer Communication and Information Technology, sinhgad Institute of Computer Science, Pandharpur, NC ${ }^{2}$ IT-2013.

[4] Sonja Grgi, Mislav Grgi, Marta Mrak, "RELIABILITY OF OBJECTIVE PICTURE QUALITY MEASURES," Journal of ELECTRICAL ENGINEERING, VOL. 55, NO. 1-2, 3-10, ISSN 1335- 3632. 2004.

[5] Department of Computing, the Hong Kong Polytechnic University (PolyU), Hyperspectral Palmprint database, PolyU, accessed on Aug. 22, 2013, available at: : http://www4.comp.polyu.edu.hk/ biometrics/Hyperspect ral Palmprint /HSP .htm. K. Elissa, "Title of paper if known," unpublished.

[6] YAN-xin SHI, CHENG Yong-mei1, “Adaptive Filter for Color Impulsive Removal Based on the HSI Color Space," 2011M. Young, The Technical Writer's Handbook. Mill Valley, CA: University Science, 1989.

[7] Huang, T.S., G.J.Yang, and G.Y.Tang. "A fast twodimensional median filtering algorithm.", IEEE transactions on Acoustics, Speech and Signal Processing, Vol ASSP 27, No. 1, February 1979

[8] Zhenhua Guo, David Zhang, Lei Zhang, and Wenhuang Liu, "Feature Band Selection for Online Multispectral Palmprint Recognition", IEEE transactions on information forensics and security, vol. 7, no. 3, June 2012.

[9] Anita G. Khandizod, R. R. Deshmukh, "Multispectral Palm print Image Fusion- A Review", International Journal of Engineering Research \& Technology (IJERT), ISSN: 2278-01s81, Vol. 3 Issue 2, February - 2014.

[10] Saruchi , "Adaptive Sigmoid Function to Enhance Low Contrast Images International Journal of Computer Applications (0975 -8887) Volume 55 - No.4, October 2012. 


\section{Appendix}

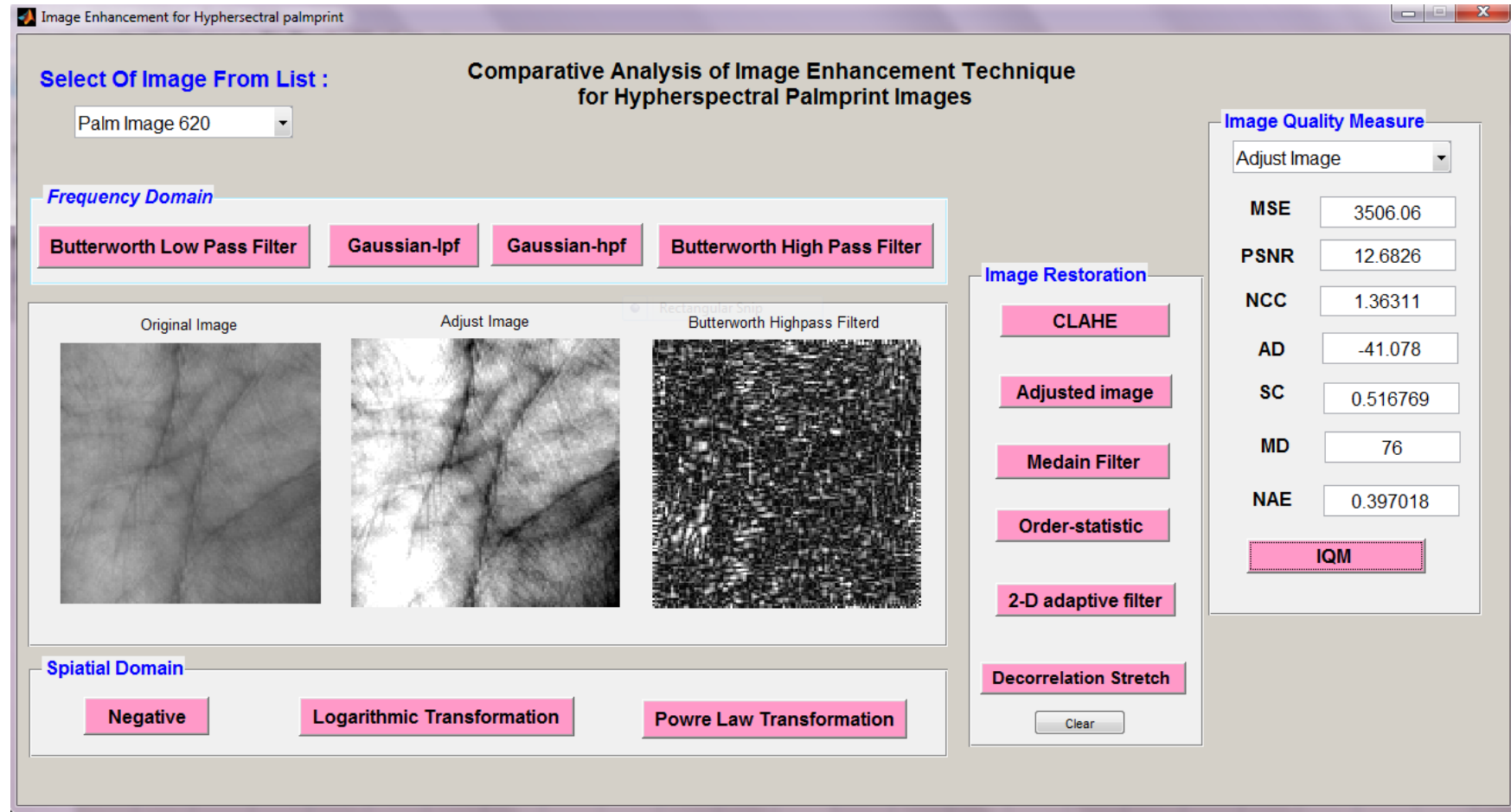

Figure. GUI (Graphical User Interface) for Hypherspectral palmprint Enhancement 